PROCEEDINGS OF THE

AMERICAN MATHEMATICAL SOCIETY

Volume 124, Number 5, May 1996

\title{
ON HANKEL OPERATORS NOT IN THE TOEPLITZ ALGEBRA
}

\author{
JOSÉ BARRÍA \\ (Communicated by Palle E. T. Jorgensen)
}

\begin{abstract}
In this paper we exhibit a class of Hankel operators, which is contained in the essential commutant of the unilateral shift, but disjoint from the Toeplitz algebra.
\end{abstract}

In [2] it is proved that if $\mathbf{E}$ is the essential commutant of the unilateral shift and $\mathbf{T}$ is the Toeplitz algebra, then the set $\mathbf{E} \backslash \mathbf{T}$ contains Hankel operators (the definitions of $\mathbf{E}$ and $\mathbf{T}$ are given below). The proof given in [2] makes use of the theory of maximal ideals of function algebras; since this method is not constructive, it does not yield concrete operators. The purpose of this paper is to exhibit a concrete class of Hankel operators which is contained in $\mathbf{E} \backslash \mathbf{T}$. The result in [2], and ours, answers one of the questions raised in [1] concerning membership in the Toeplitz algebra.

The underlying Hilbert space is $\mathbf{H}^{2}$ of the unit circle. Let $\phi$ be in $\mathbf{L}^{\infty}$ of the unit circle. The Toeplitz operator $T_{\phi}$ on $\mathbf{H}^{2}$ is defined by $T_{\varphi} f=P(\phi f)$, where $P$ is the orthogonal projection from $\mathbf{L}^{2}$ to $\mathbf{H}^{2}$. The Hankel operator $H_{\phi}$ on $\mathbf{H}^{2}$ is defined by the Hankel matrix $\left(c_{-i-j-1}\right)_{i, j=0}^{\infty}$, where $\left\{c_{n}\right\}_{-\infty}^{\infty}$ is the sequence of Fourier coefficients of $\phi$. The unilateral shift $S$ is the Toeplitz operator $T_{\phi}$ with $\phi(z)=z$. The essential commutant of $S$ is the set $\mathbf{E}$ of all operators $T$ on $\mathbf{H}^{2}$ for which $T S-S T \in \mathbf{K}$, where $\mathbf{K}$ is the ideal of all compact operators on $\mathbf{H}^{2}$. The Toeplitz algebra $\mathbf{T}$ is the $C^{*}$-algebra generated by $\left\{T_{\phi}: \phi \in \mathbf{L}^{\infty}\right\}$. The following result describes a class of Hankel operators in $\mathbf{E} \backslash \mathbf{T}$.

Theorem. Let $b$ be an infinite Blaschke product whose zero set $Z$ has its cluster points in $\{-1,1\}$. We further assume that there exists a sequence $\left\{a_{n}\right\}_{n=1}^{\infty}$ in $Z$ such that

$$
\begin{aligned}
& \lim _{n \rightarrow \infty} a_{n}=\lambda ; \\
& \lim _{n \rightarrow \infty} \frac{\left|\lambda-a_{n}\right|}{1-\left|a_{n}\right|}=+\infty .
\end{aligned}
$$

Then $H_{\bar{b}}$ is in the essential commutant of $S$, but $H_{\bar{b}}$ does not belong to the Toeplitz algebra.

The first assertion of the theorem follows from the result [2, Proposition 3.5], which we state below as a proposition. This proposition is proved in [2] using the theory of maximal ideals; the proof given below is based on function theory. First

Received by the editors September 7, 1994 and, in revised form, October 21, 1994.

1991 Mathematics Subject Classification. Primary 47B38, 47B35. 
we introduce notation and facts which are needed. For $\phi \in \mathbf{L}^{\infty}$, we write $\tilde{\phi}$ for the function defined by $\tilde{\phi}(z)=\phi(\bar{z})$. For $\phi$ and $\psi$ in $\mathbf{L}^{\infty}$ the following identities hold [1]:

$$
\begin{aligned}
T_{\phi \psi} & =T_{\phi} T_{\psi}+H_{\tilde{\phi}} H_{\psi} ; \\
H_{\phi \psi} & =H_{\phi} T_{\psi}+T_{\tilde{\phi}} H_{\psi} .
\end{aligned}
$$

For $f$ in $\mathbf{L}^{1}$ and $z$ in the open unit disk $D$, we write $P_{z}(f)$ for the Poisson integral of $f$ :

$$
P_{z}(f)=\frac{1}{2 \pi} \int_{\partial D} f P_{z} d \theta
$$

where $P_{z}$ denotes the Poisson kernel for the point $z$. We end this introduction with the algebra $\mathbf{H}^{\infty}+\mathbf{C}$, whose properties are important in our proof. Here $\mathbf{H}^{\infty}$ is the algebra of boundary functions for bounded analytic functions in $D$, and $\mathbf{C}$ is the algebra of continuous complex valued functions on $\partial D$.

Proposition. Let $b$ be a Blaschke product with zero set $Z$. Then $H_{\bar{b}}$ is in the essential commutant of $S$ if and only if $Z$ is finite or $Z$ has its cluster points in $\{-1,1\}$.

Proof. Let $A=H_{\bar{b}} S-S H_{\bar{b}}$. Applying (2) twice we obtain

$$
H_{\bar{b} z}=H_{\bar{b}} T_{z}+T_{\bar{b}} H_{z}=H_{\bar{b}} T_{z}=H_{\bar{b}} S \quad \text { and } \quad H_{\bar{z} \bar{b}}=H_{\bar{z}} T_{\bar{b}}+T_{z} H_{\bar{b}}=H_{\bar{z}} T_{\bar{b}}+S H_{\bar{b}},
$$

therefore $A=H_{(z-\bar{z}) \bar{b}}+H_{\bar{z}} T_{\bar{b}}$. Since $H_{\bar{z}}$ is of finite rank, $A$ is compact if and only if $(z-\bar{z}) \bar{b} \in \mathbf{H}^{\infty}+\mathbf{C}[7$, p. 101].

If $Z$ is finite, then $b \in \mathbf{C}$ and therefore $(\bar{z}-z) b \in \mathbf{C}$. If $Z$ has its cluster points in $\{-1,1\}$, then $b$ is continuous in $\partial D \backslash\{-1,1\}[5$, p. 68], and because $b$ is bounded it follows that $(\bar{z}-z) b \in \mathbf{C}$. In either case we have $(z-\bar{z}) \bar{b} \in \mathbf{H}^{\infty}+\mathbf{C}$.

For the converse we assume that $(z-\bar{z}) \bar{b}=f$ for some $f$ in $\mathbf{H}^{\infty}+\mathbf{C}$. We further assume that $Z$ is infinite. Let $\lambda$ be a cluster point of $Z$. Then $|\lambda|=1$. Let $a_{n} \in Z$ such that $a_{n} \rightarrow \lambda$. Since the Poisson integral is asymptotically multiplicative on $\mathbf{H}^{\infty}+\mathbf{C}\left[3\right.$, p. 169], given $\varepsilon>0$ there exists $\delta>0$ such that $\left|P_{z}(b f)-P_{z}(b) P_{z}(f)\right|<$ $\varepsilon$ for $1-|z|<\delta$. But $P_{z}(b)=b(z)$ for $z$ in $D$, and from $b f=z-\bar{z}$ on $\partial D$ we have $P_{z}(b f)=2 i \Im(z)$ for $z$ in $D$. Then $2\left|\Im\left(a_{n}\right)\right|<\varepsilon$ for $1-\left|a_{n}\right|<\delta$. This shows that $\mathfrak{I}\left(a_{n}\right) \rightarrow 0$ and therefore $\mathfrak{R}\left(a_{n}\right) \rightarrow \lambda$. Hence $\lambda$ is real and $\lambda \in\{-1,1\}$.

Lemma. Let $g \in \mathbf{H}^{\infty}+\mathbf{C}$ such that its conjugate $\bar{g}$ is also in $\mathbf{H}^{\infty}+\mathbf{C}$. Then $T T_{g}-T_{g} T \in \mathbf{K}$ for all $T$ in the Toeplitz algebra.

Proof. For $f \in \mathbf{L}^{\infty}$, let $\mathcal{A}_{f}$ be the set of all operators $T$ on $\mathbf{H}^{2}$ for which $T T_{f}-T_{f} T \in$ K. Clearly $\mathcal{A}_{f}$ is a Banach space, and from $T_{1} T_{2} T_{f}-T_{f} T_{1} T_{2}=T_{1}\left(T_{2} T_{f}-T_{f} T_{2}\right)+$ $\left(T_{1} T_{f}-T_{f} T_{1}\right) T_{2}$ it follows that $\mathcal{A}_{f}$ is a Banach algebra. Now we consider a function $g$ satisfying the hypothesis of the lemma. Since $H_{g}$ is compact [7, p. 101], from (1) we conclude that $T_{\phi g}-T_{\phi} T_{g} \in \mathbf{K}$ for all $\phi$ in $\mathbf{L}^{\infty}$. The same argument applied to $\bar{\phi}$ and $\bar{g}$ gives that $T_{\bar{\phi} \bar{g}}-T_{\bar{\phi}} T_{\bar{g}} \in \mathbf{K}$ and by taking the adjoint we conclude that $T_{\phi g}-T_{g} T_{\phi} \in \mathbf{K}$. Therefore $T_{\phi} T_{g}-T_{g} T_{\phi} \in \mathbf{K}$ for all $\phi$ in $\mathbf{L}^{\infty}$. Since we can interchange the roles of $g$ and $\bar{g}$, the set $\left\{T_{\phi}: \phi \in \mathbf{L}^{\infty}\right\}$ is contained in $\mathcal{A}_{g} \cap \mathcal{A}_{\bar{g}}$. Because $\mathcal{A}_{g} \cap \mathcal{A}_{\bar{g}}$ is closed under the adjoint operation, $\mathcal{A}_{g} \cap \mathcal{A}_{\bar{g}}$ is a $C^{*}$-algebra, and therefore $\mathbf{T} \subset \mathcal{A}_{g} \cap \mathcal{A}_{\bar{g}}$. 
Now to prove the second part of the theorem it is enough to exhibit a function $g$ satisfying the hypothesis of the lemma, for which the operator $H_{\bar{b}} T_{g}-T_{g} H_{\bar{b}}$ is not compact. The construction of $g$ will be carried out in the Banach space BMO of functions of bounded mean oscillation. For the definition and properties of this space we refer the reader to [7]. A key part in the construction of $g$ is played by the following result [4, Lemma 1].

Proposition. Let $I$ be a subarc of $\partial D$ and $J$ a subarc of $I$ with the same center. There is a continuous function $u$ with values in $[0,1]$ such that $u=1$ on $J, u=0$ off $I$, and $\|u\|_{\mathrm{BMO}} \leq \mathrm{const} / \log (|I| /|J|)$.

Here, $|I|$ denotes the length of $I$, and \|\|$_{\text {BMO }}$ is the norm in the space BMO.

Construction of a function. Now we are ready to construct a particular realvalued function $g$ in $\mathbf{H}^{\infty}+\mathbf{C}$. By hypothesis we have the sequence $\left\{a_{n}\right\}_{n=1}^{\infty}$ in $Z$ satisfying conditions (i) and (ii), and $\lambda \in\{-1,1\}$. We write $a_{n}=\left|a_{n}\right| e^{i \theta_{n}}$, with $-\pi<\theta_{n} \leq \pi$. By (ii) we may assume that $\theta_{n} \neq 0$ and $\theta_{n} \neq \pi$ for all $n$. For $a=|a| e^{i \theta}$ in $D$ we have the identity

$$
\frac{|\lambda-a|^{2}}{(1-|a|)^{2}}=1+2|a| \cdot \frac{1-\lambda \cos \theta}{(1-|a|)^{2}}
$$

Then from (ii) it follows that

$$
\lim _{n \rightarrow \infty} \frac{1-\lambda \cos \theta_{n}}{\left(1-\left|a_{n}\right|\right)^{2}}=+\infty
$$

For the rest of the construction we assume that the set $\left\{n: \theta_{n}>0\right\}$ is infinite. (If $\theta_{n}<0$ for all $n$, then the modifications that are necessary are obvious.) Then from (i) it follows that $\theta_{n} \rightarrow 0$ (if $\lambda=1$ ) or $\theta_{n} \rightarrow \pi$ (if $\lambda=-1$ ). First we consider the case $\lambda=1$. Since $\lim _{\theta \rightarrow 0} \frac{1-\cos \theta}{\theta^{2}}=\frac{1}{2}$, from (iii) it follows that

$$
\lim _{n \rightarrow \infty} \frac{\theta_{n}}{1-\left|a_{n}\right|}=+\infty
$$

Then we can choose a subsequence $\left\{a_{n_{k}}\right\}_{k=1}^{\infty}$ of $\left\{a_{n}\right\}_{n=1}^{\infty}$ such that

$$
2 \pi \exp \left(2^{k}\right)<\frac{\theta_{n_{k}}}{1-\left|a_{n_{k}}\right|} \text { and } 3 \theta_{n_{k+1}}<\theta_{n_{k}} \quad \text { for } k=1, \ldots, \infty .
$$

Let $I_{k}$ be the subarc of $\partial D$ with center $e^{i \theta_{n_{k}}}$ and length $\theta_{n_{k}}$. From the second inequality it follows that $I_{k} \cap I_{l}=\emptyset$ for $k \neq l$. Let $J_{k}$ be the subarc of $I_{k}$ with the same center as $I_{k}$ and whose length is $2 \pi\left(1-\left|a_{n_{k}}\right|\right)$. Then $\left|J_{k}\right| \exp \left(2^{k}\right)<\left|I_{k}\right|$, and therefore $1 / \log \left(\left|I_{k}\right| /\left|J_{k}\right|\right)<2^{-k}$ for $k \geq 1$. Now we apply the proposition above to $I_{k}$ and $J_{k}$ to obtain a continuous function $u_{k}$ with values in $[0,1]$ such that $u_{k}=1$ on $J_{k}, u_{k}=0$ off $I_{k}$, and $\left\|u_{k}\right\|_{\mathrm{BMO}} \leq$ (const) $2^{-k}$. Let $u=\sum_{k=1}^{\infty} u_{k}$. The series converges in BMO and the terms are continuous.

Then from [7, p. 49], $u$ can be written as $u=f+v$ where $f$ is in $\mathbf{C}$ and $v$ is the harmonic conjugate of a function $h$ in $\mathbf{C}$. Then $h+i v$ is analytic in $D$, and since $u$ is bounded $\left(\|u\|_{\infty}=1\right)$, it follows that $h+i v \in \mathbf{H}^{\infty}$. Since $f+i h \in \mathbf{C}$, the equality $u=-i(h+i v)+(f+i h)$ shows that $u \in \mathbf{H}^{\infty}+\mathbf{C}$. Now we define $g=u-\tilde{u}$. Then $g$ is a real-valued function and $g \in \mathbf{H}^{\infty}+\mathbf{C}$. So $g$ satisfies the hypothesis of the lemma. 
Finally, we consider the case $\lambda=-1$. Then (iii) becomes

$$
\lim _{n \rightarrow \infty} \frac{1-\cos \left(\pi-\theta_{n}\right)}{\left(1-\left|a_{n}\right|\right)^{2}}=+\infty,
$$

and from this it follows that

$$
\lim _{n \rightarrow \infty} \frac{\pi-\theta_{n}}{1-\left|a_{n}\right|}=+\infty
$$

Now one gets inequalities similar to (iv), where $\theta_{n_{k}}$ is replaced by $\pi-\theta_{n_{k}}$. Also, in the definition of the subarc $I_{k}$ we need to change the length (but not the center) to $\pi-\theta_{n_{k}}$. Then the construction of $g$ proceeds as above.

Last part of the theorem. For the function $g$ constructed above we show that $H_{\bar{b}} T_{g}-T_{g} H_{\bar{b}}$ is not compact. From this and the lemma it will follow that $H_{\bar{b}}$ is not in the Toeplitz algebra. Let $A=H_{\bar{b}} T_{g}-T_{g} H_{\bar{b}}$. Applying (2) to $H_{\bar{b} g}$ and $H_{\tilde{g} \bar{b}}$ (as we did in the proof of the first proposition) we obtain

$$
A=H_{(g-\tilde{g}) \bar{b}}+H_{\tilde{g}} T_{\bar{b}}-T_{\tilde{\bar{b}}} H_{g} .
$$

Since $g$ and $\tilde{g}$ are in $\mathbf{H}^{\infty}+\mathbf{C}, H_{g}$ and $H_{\tilde{g}}$ are compact, and therefore $A$ is not compact if and only if $(g-\tilde{g}) \bar{b}$ does not belong to $\mathbf{H}^{\infty}+\mathbf{C}$. To arrive at a contradiction, let us assume that $(g-\tilde{g}) \bar{b}=f$ for some $f \in \mathbf{H}^{\infty}+\mathbf{C}$. Then $g-\tilde{g}=b f$. From the definition of $g$ it follows that $\tilde{g}=-g$, so $b f=2 g$. Since the Poisson integral is asymptotically multiplicative on $\mathbf{H}^{\infty}+\mathbf{C}$, there exists $\delta>0$ such that

$$
\left|P_{z}(b f)-P_{z}(b) P_{z}(f)\right|<1 \text { for } 1-|z|<\delta .
$$

Therefore, $\left|P_{a_{n_{k}}}(g)\right|<\frac{1}{2}$ for all $k$ 's in the complement of a finite set. Now a contradiction will be obtained by showing that $P_{a_{n_{k}}}(g) \rightarrow 1$ as $k \rightarrow \infty$. For this we use a result in [6] concerning the Poisson integral and certain averaging functionals. For $z \neq 0$ in $D$, we let $I_{z}$ denote the closed subarc of $\partial D$ whose center is $z /|z|$ and whose length is $2 \pi(1-|z|)$. Then from [6, Lemma 5] applied to $g$, we conclude that given $\varepsilon>0$, there exists $\delta^{\prime}>0$ such that

$$
\left|P_{z}(g)-\frac{1}{\left|I_{z}\right|} \int_{I_{z}} g\left(e^{i \theta}\right) d \theta\right|<\varepsilon \text { for } 1-|z|<\delta^{\prime} .
$$

But from the definition of $J_{k}$ and $I_{z}$, with $z=a_{n_{k}}$, we have $I_{a_{n_{k}}}=J_{k}$. Also, from the definition of $g$, because the functions $u_{k}$ have disjoint supports, we have

$$
\int_{J_{k}} g\left(e^{i \theta}\right) d \theta=\int_{J_{k}} u_{k}\left(e^{i \theta}\right) d \theta=\int_{J_{k}} 1 d \theta=\left|J_{k}\right| .
$$

Then, from the above inequality, $\left|P_{a_{n_{k}}}(g)-1\right|<\varepsilon$ for $1-\left|a_{n_{k}}\right|<\delta^{\prime}$. Now we have obtained the desired contradiction, and therefore $A$ is not compact. The proof of the theorem is complete.

\section{ACKNOWLEDGMENT}

The results in this paper were obtained while the author was on Sabbatical leave from Santa Clara University and a visitor at the University of California, Berkeley. He would like to thank Donald Sarason for helpful conversations. 


\section{REFERENCES}

1. J. Barría and P. R. Halmos, Asympototic Toeplitz operators, Trans. Amer. Math. Soc. 273 (1982), 621-630. MR 84j:47040

2. X. Chen and F. Chen, Hankel operators in the set of essential Toeplitz operators Acta Math. Sinica 6 (1990), 354-363. MR 91k:47056

3. R. G. Douglas, Banach algebra techniques in operator theory, Academic Press, New York, 1972. MR 50:14335

4. C. Guillory and D. Sarason, The algebra of quasicontinuous functions, Proc. Roy. Irish Acad. 84 (1984), 57-67. MR 86j:46055

5. K. Hoffman Banach spaces of analytic functions, Prentice-Hall, Englewood Cliffs, NJ, 1962. MR 24:A2844

6. D. Sarason, Toeplitz operators with piecewise quasicontinuous symbols, Indiana Univ. Math. J. 26 (1977) 817-838. MR 57:3906

7. Blacksburg, VA, 1978. MR 80d:30035

Department of Mathematics, Santa Clara University, Santa Clara, California 95053

E-mail address: jbarria@scuacc.scu.edu 